\title{
P022: Antibiograms of consecutive urinary tract samples in elderly
}

\author{
K Latour ${ }^{1}$, B Jans ${ }^{2}$, S Coenen ${ }^{3}$, R Preal $^{4}$, B Catry ${ }^{*}$ \\ From 2nd International Conference on Prevention and Infection Control (ICPIC 2013) \\ Geneva, Switzerland. 25-28 June 2013
}

\section{Introduction}

Urinary tract infections are the main indication for antimicrobials in elderly.

\section{Objectives}

Despite the treat for resistance dissemination and therapy failure, clinicians are seldom informed on the per patient evolution of antimicrobial resistance in pathogens.

\section{Methods}

Laboratory results were obtained from 13 voluntary diagnostic laboratories (12 hospital-associated) in Belgium during the year 2005. Susceptibility profiles were done by Kirby Bauer disk diffusion according to CLSI. The first two urine samples from patients older than 65 year were included.

\section{Results}

Following organisms were predominantly isolated ( $\mathrm{N}$ first samples/N second samples): E. coli (7188/1654), E. faecalis (1282/403), P. mirabilis (1230/313), K. pneumonia (673/ 173), P. aeruginosa (293/120), E. aerogenes (375/203), S. aureus (158/54), M. morganii (347/89), Group B streptococci $(149 / 31)$, C. freundii complex $(101 / 29)$. When comparing first versus second samples antibiograms for E. coli, a decrease in susceptibility was found for the following antimicrobial agents: cotrimoxazole $-6.9 \%$; nitrofurantoin $-2.8 \%$, fosfomycin $0.0 \%$; ciprofloxacin $-10.8 \%$; cefuroxime $-5.6 \%$; amoxicillin-clavulanic acid -5.6\%; ampicillin -10.5\%. For E. faecalis, marked decreases were found for nitrofurantoin $-2.4 \%$; fosfomycin $-2.2 \%$; -ciprofloxacin -10.3\%; and only mild decreases for amoxicillin-clavulanic acid $0.0 \%$; and ampicillin $-1.2 \%$. For K. pneumoniae decreases were in the range of -2.9 to $-4.1 \%$ for cotrimoxazole, ciprofloxacine, cefuroxime and amoxicillin-clavulanic acid, and was $-12.4 \%$ for nitrofurantoin. For $S$. aureus and C. freundii no decrease $(<-0.1 \%)$ was seen for nitrofurantoin and fosfomycin. For E. aerogenes, decreases of -18 and $-12.5 \%$ were found for cotrimoxazole and fosfomycin, respectively. M. morganii showed in consecutive samples less susceptibility for cotrimoxazole $(-16.2 \%)$, fosfomycine $(-13.0 \%)$ and ciprofloxacin $(-10.5 \%)$, while only a marginal decrease was found for nitrofurantoin $(-0.5 \%)$.

\section{Conclusion}

The resistance selection influence of consecutive samples depends on the antibiotic-bacterium combinations, and thus might be taken into account when empiric therapy guidelines for urinary tract infections in elderly are reviewed.

\section{Disclosure of interest}

None declared.

\section{Author details}

${ }^{1}$ Healthcare Associated Infections (NSIH), Brussels, Belgium. ${ }^{2}$ Scientific Institute of Public Health, Brussels, Belgium. ${ }^{3}$ University of Antwerp, Wilrijk, Brussels, Belgium. ${ }^{4}$ Intermutualistic Agency, Brussels, Belgium.

Published: 20 June 2013

\section{doi:10.1186/2047-2994-2-S1-P22}

Cite this article as: Latour et al:: P022: Antibiograms of consecutive urinary tract samples in elderly. Antimicrobial Resistance and Infection Control 2013 2(Suppl 1):P22. 